\section{Prevalência e fatores associados à anemia entre crianças atendidas em creches públicas de São Paulo}

\author{
Prevalence and associated factors \\ for anemia among children of public \\ day-care centers in the city São \\ Paulo
}

Milena Baptista Bueno

Soraya Sant'Ana de Castro Selem

José Alfredo Gomes Arêas

Regina Mara Fisberg

Departamento de Nutrição da Faculdade de Saúde Pública da Universidade de São Paulo.

Pesquisa financiada pela Fundação de Amparo a Pesquisa do Estado de São Paulo (FAPESP) processo 98/08095-9

Correspondência: Milena Baptista Bueno. Departamento de Nutrição/Faculdade de Saúde Pública/USP. Av.Dr Arnaldo, 715 - São Paulo - SP CEP 01246-904.E-mail: mibueno@usp.br

\section{Resumo}

Um dos grandes desafios na saúde pública é o combate à anemia ferropriva em crianças. Considerando a importância da cidade de São Paulo em relação ao tamanho da sua população e a escassez de estudos representativos de crianças que freqüentam creches, este estudo teve como objetivo analisar a prevalência de anemia em uma amostra probabilística de crianças freqüentadoras de creches da rede municipal de São Paulo e identificar os fatores associados. Este é um estudo transversal com 330 pré-escolares sorteados entre 20 creches públicas. Anemia foi definida como hemoglobina menor de 11g/dL. Utilizou-se regressão logística hierarquizada para análise múltipla. A prevalência de anemia foi de $68,8 \%\left(\mathrm{IC}_{95 \%}=63,8 \% ; 73,8 \%\right)$. Entre as crianças com anemia, o percentual de anemia grave $(<9,5 \mathrm{~g} / \mathrm{dL})$ foi de $26,9 \%$. Estiveram associados à anemia idade menor de 24 meses (Odds ratio $(\mathrm{OR})=$ 2,$\left.7 ; \mathrm{IC}_{95 \%}=1,4 ; 5,2\right)$ e idade do responsável pela criança menor de 25 anos $(\mathrm{OR}=1,8$; $\left.\mathrm{IC}_{95 \%}=1,0 ; 3,2\right)$. A prevalência de anemia foi alta quando comparada a outros estudos. O acompanhamento de programas de combate a esta doença deve ser realizado e modificado, quando necessário, especialmente entre crianças freqüentadoras de creches públicas menores de dois anos que possuem responsáveis mais jovens.

Palavras-chaves: Anemia. Creche. Pré-escolares. 


\section{Abstract}

Iron deficiency anemia in children is a challenge for public health. Considering that São Paulo is the largest city in Brazil and the lack of studies with representative samples of children enrolled in public day-care centers, a cross-sectional study was conducted in the city of São Paulo to estimate the prevalence of anemia and to identify factors associated with the disease. A random sample of 330 preschool children of 20 public day cares centers was analyzed. Anemia was defined as hemoglobin below $11.0 \mathrm{~g} /$ dL. A hierarchical logistic regression model was applied. The prevalence of anemia was $68.8 \%\left(\mathrm{CI}_{95 \%}=63.8 \%\right.$; $\left.73.8 \%\right)$. Factors associated with anemia were child's age below 24 months (Odds Ratio (OR): 2.7; $\mathrm{CI}_{95 \%}=1.4$; 5.2) and age of the child's guardian below 25 years of age (OR: 1.8; $\left.\mathrm{IC}_{95 \%}=1.0 ; 3.2\right)$. The prevalence of anemia was higher than in most studies performed in different cities of Brazil. Programs for anemia control should be monitored and modified, if necessary, especially for children in public daycare centers below two years of age and with young guardians.

Keywords: Anemia. Day care centers. Preschool.

\section{Introdução}

A anemia é considerada a doença mais prevalente no mundo, especialmente a caracterizada por deficiência da ingestão de ferro. Constitui-se no distúrbio nutricional usualmente mais freqüente na infância, independente do desenvolvimento econômico do país. No Brasil, distinguese de outras modalidades de deficiência nutricional, pois a tendência da prevalência da anemia tem sido de aumento ${ }^{1,2}$.

A redução da concentração de hemoglobina sanguínea tem como principais sinais e sintomas alterações da pele e mucosas, alterações gastrintestinais, fadiga, fraqueza, palpitação, redução da função cognitiva, do crescimento e do desenvolvimento psicomotor, entre outros. Entretanto, os mecanismos homeostáticos fornecem adaptação, podendo-se também encontrar anemia em indivíduos que não apresentam qualquer sintoma ${ }^{3}$.

Nos últimos anos, estudos realizados por todo o Brasil mostraram prevalência de anemia em crianças de 6 meses a 5 anos variando de $36,4 \%$ a $63,0 \% \%^{2,4-7}$. Especificamente na cidade de São Paulo, Monteiro e col. ${ }^{1}$ verificaram prevalência de $46,9 \%$ de anemia em crianças de 0 a 59 meses em 1995/96.

Considerando a importância da cidade de São Paulo em relação ao tamanho de sua população e à escassez de estudos representativos de crianças que freqüentam creches, instituições destinadas ao atendimento de crianças na fase em que estão mais suscetíveis ao surgimento de anemia ferropriva, este estudo teve como objetivo analisar a prevalência de anemia em uma amostra probabilística de crianças freqüentadoras de creches da rede municipal de São Paulo e identificar os fatores associados.

\section{Metodologia}

Os dados analisados no presente estudo originaram-se de uma coorte de crianças que freqüentavam creches públicas do 
município de São Paulo, que teve início em 2000, com o objetivo principal de verificar a evolução nutricional ${ }^{8}$. Na última observação, além dos dados antropométricos coletou-se uma amostra de sangue para avaliar o estado nutricional em relação ao ferro. A coleta da amostra de sangue, assim como os outros dados utilizados neste estudo, foi realizada entre março e abril de 2001.

Em 2000, as creches públicas do município de São Paulo atendiam aproximadamente 80.000 crianças, na faixa etária de 4 a 83 meses, distribuídas em 718 unidades. Em todas elas, o atendimento era em período integral. Para o cálculo do tamanho da amostra, considerou-se prevalência de anemia de $47 \%^{1}$ e admitiu-se como aceitável uma precisão tal que, em 95\% das possíveis amostras, o erro máximo de amostragem não ultrapassasse $5 \%$.

$\mathrm{O}$ procedimento de amostragem foi por conglomerados em duas etapas. Para garantir a representatividade das regiões do município de São Paulo, o sistema de referência para a primeira etapa de amostragem foi ordenado segundo regiões administrativas. Utilizando-se um procedimento de amostragem sistemática obteve-se uma amostra de 20 creches estratificadas por região. As crianças a serem avaliadas nas creches amostradas foram selecionadas por sorteio sistemático, depois de calculada a fração global da amostra.

Realizou-se entrevista com os responsáveis pela criança utilizando um questionário contendo informações referentes à identificação da criança e da família (dados econômicos, sociais e demográficos) e de diarréia referida nos últimos 30 dias. Os dados analisados referentes ao responsável pela criança são, em $90 \%$ dos casos, os dados da mãe da criança, os $10 \%$ restantes referem-se a responsáveis com outro grau de parentesco como avó/avô, pai, irmãos, entre outros.

A padronização das medidas antropométricas seguiu as recomendações de Lohman e col. ${ }^{9}$ e foi realizada no laboratório do Núcleo de Pesquisas Epidemiológicas em Nutrição e Saúde da Universida- de de São Paulo (NUPENS/USP). Para descrever o estado nutricional, foram utilizados os valores de escore-Z de altura para idade, tendo como distribuição de referência o padrão do National Center for Health Statistics - NCHS.

A amostra de sangue foi colhida por meio de punção digital com lancetas descartáveis por estudantes de graduação e pós-graduação, previamente treinados. A dosagem de concentração de hemoglobina foi determinada pelo hemoglobinômetro portátil HemoCue. Para o diagnóstico de anemia foi utilizado o critério recomendado pela Organização Mundial da Saúde (OMS), que propõe $11 \mathrm{~g} / \mathrm{dL}$ como valor crítico de concentração de hemoglobina para crianças a partir de 6 meses e abaixo de 9,5 g/dL como anemia grave ${ }^{10}$. Crianças menores de 6 meses ou que estavam utilizando sulfato ferroso no dia da coleta foram excluídas.

A análise estatística incluiu regressão logística múltipla com modelo de seleção hierarquizada de variáveis. O modelo conceitual adotado, como mostra a Figura 1 , foi o proposto por Silva e col. ${ }^{2}$.

A partir deste modelo conceitual, foram testadas as associações de cada variável com a anemia, incluindo-se no modelo hierárquico apenas as que mostraram $\mathrm{p}<0,20$ na análise univariada. A seguir foi realizada a análise múltipla para determinação dos fatores associados. No modelo, foram mantidas as variáveis com valor de $\mathrm{p}<0,05$. Os dados foram digitados e tratados estatisticamente utilizando-se os softwares EPIINFO versão 2000 e STATA v.8.0.

A concordância para participar da pesquisa foi feita por escrito, com assinatura do termo de consentimento livre e esclarecido pelos responsáveis da criança. Este estudo foi aprovado pelo Comitê de Ética em Pesquisa em Seres Humanos da Faculdade de Saúde Pública/USP. As crianças identificadas como anêmicas foram encaminhadas para as unidades básicas de saúde da região para o adequado tratamento. Esta pesquisa não apresenta conflito de interesse real ou potencial. 
1. Dimensão dos processos estruturais da sociedade (Bloco socioeconômico):

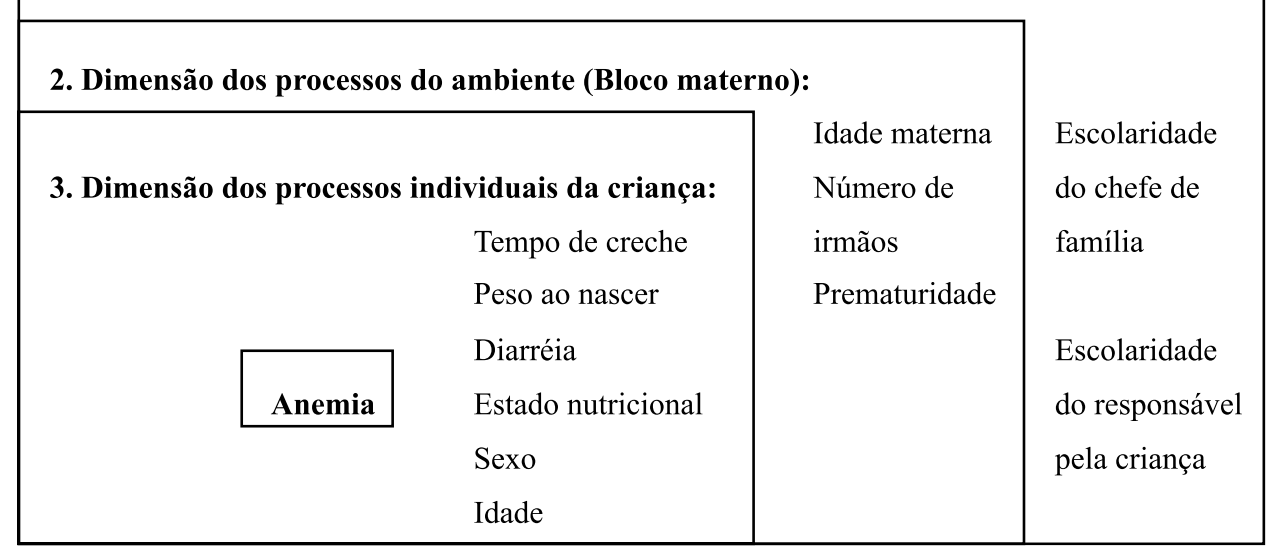

Figura 1- Modelo conceitual com seleção hierárquica

Figure 1- Conceptual model for hierarchical analysis

\section{Resultados}

A amostra foi composta por 330 crianças com idade entre 6 e 75 meses, sendo a média 35,3 meses ( $\mathrm{DP}=15,2$ meses). A distribuição entre os sexos foi homogênea $(49,7 \%$ masculino e $50,3 \%$ feminino). A média de tempo de freqüência na creche foi de 22,7 meses ( $\mathrm{DP}=10,7$ meses).

A incidência acumulada de baixo peso ao nascer (< 2500 gramas) foi de $12,3 \%$ e de prematuridade $10,7 \%$. Somente 3 crianças apresentaram muito baixo peso ao nascer (< 1500gramas). Todos os prematuros nasceram com peso adequado à idade gestacional. A incidência de diarréia, nos últimos 30 dias, relatada pelos responsáveis pela criança, foi de $12,7 \%$. A média de irmãos das crianças era de 1,34 (DP = 1,35), sendo $33,1 \%$ primeiro filho.

A média de escolaridade do responsável e do chefe de família era de 6,7 anos (DP = 3,1 anos) e 6,1 anos ( DP = 3,3 anos), respectivamente. A média de idade dos responsáveis pela criança era de 29,2 anos (DP $=7,4$ anos), sendo a maioria casada (63,1\%). Em 85 famílias (25\%), o chefe de família e o responsável pela criança eram a mesma pessoa. Destes, $71,8 \%$ era a mãe da criança que acumulava as responsabi-

Tabela 1- Distribuição de crianças freqüentadoras de creches segundo região do município e anemia. São Paulo, 2001.

Table 1- Distribution of children in public day-care centers by region and anemia. São Paulo, 2001.

\begin{tabular}{lllll}
\hline Região do município de São Paulo & \multicolumn{4}{l}{ Anemia } \\
\cline { 2 - 5 } & Não & & Sim & \\
\cline { 2 - 5 } & $\mathrm{n}$ & $\%$ & $\mathrm{n}$ & $\%$ \\
\hline Noroeste & 14 & 26,9 & 38 & 73,1 \\
Nordeste & 17 & 28,1 & 42 & 71,2 \\
Sul & 30 & 30,9 & 67 & 69,1 \\
Leste & 19 & 33,3 & 38 & 66,7 \\
Sudeste & 12 & 48,0 & 13 & 52,0 \\
Oeste & 6 & 33,3 & 12 & 66,7 \\
\hline Total & 103 & 31,2 & 227 & 68,8 \\
\hline${ }^{*} p>0,05$ & & & &
\end{tabular}


lidades pelo cuidado infantil e manutenção financeira do lar.

A prevalência de anemia observada foi de $68,8 \%\left(\mathrm{IC}_{95 \%}: 63,76-73,81\right)$, dos quais $26,9 \%$ apresentavam anemia grave, o que significa que, a cada quatro crianças anêmicas, uma é anêmica grave. Não houve diferença estatística entre prevalência de anemia e região do município que se encontrava a instituição, como mostra a Tabela 1 .

A Tabela 2 mostra a prevalência de anemia entre as categorias das variáveis estudadas e a análise bivariada. No modelo final da análise de regressão logística múltipla hierarquizada (Tabela 3) permanece-

Tabela 2 - Prevalência de anemia das crianças frequentadoras das creches. São Paulo, 2001.

Table 2 - Prevalence of anemia among children in public day-care centers. São Paulo, 2001.

\begin{tabular}{|c|c|c|c|c|c|}
\hline \multirow[t]{2}{*}{ Variáveis } & \multirow{2}{*}{$\begin{array}{l}\text { Total } \\
\mathrm{n}\end{array}$} & \multicolumn{2}{|c|}{ Prevalência de Anemia } & \multirow{2}{*}{$\begin{array}{l}\text { Razão de } \\
\text { prevalências }\end{array}$} & \multirow[t]{2}{*}{$\mathrm{IC}_{95 \%}$} \\
\hline & & $\mathrm{n}$ & $\%$ & & \\
\hline \multicolumn{6}{|c|}{ Escolaridade do responsável (anos) } \\
\hline$\leq 4$ & 70 & 47 & 67,1 & 0,93 & $0,46-1,85$ \\
\hline $4-\mid 8$ & 167 & 117 & 70,1 & 1,06 & $0,59-1,90$ \\
\hline$>8$ & 77 & 53 & 68,8 & 1 & \\
\hline \multicolumn{6}{|c|}{ Escolaridade do chefe (anos) } \\
\hline$\leq 4$ & 78 & 59 & 75,6 & 1,16 & $0,53-2,56$ \\
\hline $4-\mid 8$ & 90 & 60 & 66,7 & 0,75 & $0,36-1,57$ \\
\hline$>8$ & 55 & 40 & 72,7 & 1 & \\
\hline \multicolumn{6}{|c|}{ Idade do responsável (anos)* } \\
\hline$\leq 25$ & 111 & 83 & 74,8 & 1,89 & $1,09-3,27$ \\
\hline $25-30$ & 78 & 58 & 74,4 & 1,85 & $1,01-3,41$ \\
\hline$>30$ & 141 & 86 & 61,0 & 1 & \\
\hline \multicolumn{6}{|c|}{ Sexo da criança* } \\
\hline Feminino & 164 & 106 & 63,9 & 1 & \\
\hline Masculino & 166 & 121 & 73,8 & 1,59 & $0,99-2,55$ \\
\hline \multicolumn{6}{|c|}{ Idade da criança (meses) ${ }^{* *}$} \\
\hline$<24$ & 83 & 69 & 83,1 & 2,78 & $1,48-5,21$ \\
\hline$\geq 24$ & 247 & 158 & 64,0 & 1 & \\
\hline \multicolumn{6}{|c|}{ Tempo da criança na creche (meses) } \\
\hline$<15$ & 92 & 70 & 76,1 & 1,81 & $1,00-3,27$ \\
\hline $15 \mid-25$ & 100 & 69 & 69,0 & 1,26 & $0,73-2,19$ \\
\hline$\geq 25$ & 138 & 88 & 63,8 & 1 & \\
\hline \multicolumn{6}{|l|}{ Prematuridade } \\
\hline Não & 284 & 200 & 70,4 & 1 & \\
\hline Sim & 34 & 19 & 55,9 & 1,88 & $0,91-3,87$ \\
\hline \multicolumn{6}{|c|}{ Peso ao nascer (g) } \\
\hline$<2500$ & 38 & 23 & 60,5 & 0,62 & $0,30-1,21$ \\
\hline $2500 \mid-3000$ & 73 & 46 & 63,0 & 0,69 & $0,39-1,21$ \\
\hline$\geq 3000$ & 198 & 158 & 71,2 & 1 & \\
\hline \multicolumn{6}{|c|}{ Número de irmãos } \\
\hline 0 & 106 & 74 & 69,8 & 1 & \\
\hline $1 \mid-3$ & 155 & 110 & 71,0 & 1,06 & $0,61-1,81$ \\
\hline$\geq 3$ & 56 & 35 & 62,5 & 0,72 & $0,36-1,42$ \\
\hline \multicolumn{6}{|l|}{ Diarréia } \\
\hline Não & 287 & 197 & 68,6 & 1 & \\
\hline Sim & 42 & 30 & 70,0 & 1,14 & $0,56-2,33$ \\
\hline \multicolumn{6}{|c|}{ Escore-Z de altura para idade } \\
\hline$<-2$ & 21 & 19 & 90,5 & 3,91 & $0,86-17,8$ \\
\hline$-2 \mid--1,0$ & 15 & 9 & 60,0 & 1,12 & $0,53-3,32$ \\
\hline$\geq-1,0$ & 294 & 199 & 67,7 & 1 & \\
\hline
\end{tabular}

${ }^{*} p<0,05 ; * * p<0,01$ 
Tabela 3 - Análise de regressão logística múltipla hierarquizada para fatores associados à anemia em crianças freqüentadoras de creches. São Pualo, 2001.

Table 3 - Multivariate hierarchical analyis for associated factors for anemia among children in public day-care centers. São Paulo, 2001.

\begin{tabular}{|c|c|c|c|c|c|c|c|}
\hline \multirow[t]{2}{*}{ Variáveis } & \multicolumn{2}{|c|}{ 1a Etapa } & \multicolumn{2}{|c|}{$2^{\text {a Etapa }}$} & \multicolumn{3}{|c|}{ 3a Etapa } \\
\hline & $R P^{*}$ & $\mathrm{IC}_{95 \%}$ & $R P$ & $\mathrm{IC}_{95 \%}$ & $R P$ & $\mathrm{IC}_{95 \%}$ & \\
\hline \multicolumn{8}{|c|}{ Idade do responsável (anos) } \\
\hline$\leq 25$ & 1,94 & $1,11-3,39$ & 1,86 & $1,03-3,34$ & 1,84 & $1,06-3,21$ & \\
\hline $25-30$ & 1,83 & $0,98-3,41$ & 1,90 & $0,99-3,64$ & 1,84 & $0,99-3,42$ & \\
\hline$>30$ & 1 & & 1 & & 1 & & \\
\hline \multicolumn{8}{|l|}{ Prematuridade } \\
\hline Sim & 0,55 & $0,26-1,14$ & Excl & - & - & - & \\
\hline Não & 1 & & & - & - & - & \\
\hline \multicolumn{8}{|c|}{ Tempo da criança na creche (meses) } \\
\hline$<15$ & - & - & 1,89 & $0,98-3,64$ & Excl & - & \\
\hline $15 \mid-25$ & - & - & 1,40 & $0,78-2,49$ & & - & \\
\hline$\geq 25$ & - & - & 1 & & & - & \\
\hline \multicolumn{8}{|c|}{ Peso ao nascer (g) } \\
\hline$<2500$ & - & - & 0,49 & $0,23-1,04$ & Excl & - & \\
\hline $2500 \mid-3000$ & - & - & 0,62 & $0,35-1,12$ & & - & \\
\hline$\geq 3000$ & - & - & 1 & & & - & \\
\hline \multicolumn{8}{|l|}{ Escore-Z de A/I } \\
\hline$<-1$ & - & - & 1,88 & $0,99-3,57$ & Excl & - & \\
\hline$\geq-1$ & - & - & 1 & & & - & \\
\hline \multicolumn{8}{|c|}{ Idade da criança (meses) } \\
\hline$<24$ & - & - & - & - & 2,73 & $1,45-5,15$ & \\
\hline$\geq 24$ & - & - & - & - & 1 & & \\
\hline Sexo da criança & - & - & - & - & & & \\
\hline Masculino & - & - & - & - & 1,53 & $0,94-2,48$ & Excl \\
\hline Feminino & - & - & - & - & 1 & & \\
\hline
\end{tabular}

Excl. = excluídos ( $\mathrm{p}$ de Wald $>0,05) /$ Excl. $=$ excluded $($ Wald $\mathrm{p}>0.05)$

* $\mathrm{RP}=$ Razão de Prevalências / $\mathrm{RP}=$ Prevalence ratio

ram idade do responsável pela criança e idade da criança

\section{Discussão}

A prevalência de anemia foi alta nas crianças das duas faixas etárias estudadas (menor de 24 meses e maior ou igual a 24 meses) quando comparada aos resultados encontrados em outros estudos realizados no Brasil que utilizaram o mesmo critério de diagnóstico utilizado nesta pesqui$\mathrm{sa}^{1,2,5,7}$.

Silva et al. ${ }^{2}$ (2001), avaliando crianças de 0 a 36 meses que freqüentavam escolas municipais de Porto Alegre, a maioria em período integral, observaram prevalência de anemia de 47,8\%. Almeida e col. ${ }^{11}$ veri- ficaram prevalência de anemia de 62,5\% entre crianças de 12 a 72 meses em duas creches da cidade de Pontal, e Brunken e col. ${ }^{4}$ observaram prevalência de $63 \%$ no universo de crianças menores de 36 meses na cidade de Cuiabá, valores mais próximos da prevalência observada no presente trabalho.

Corroborando com outros estudos, a prevalência de anemia foi maior entre os menores de 24 meses, conhecido fator de risco para anemia. Justifica-se este resultado pela maior velocidade de crescimento em crianças nessa faixa etária, desmame precoce, maior prevalência de doenças, atraso na introdução de alimentos ricos em ferro e dieta monótona ${ }^{2}$. Destacase que, por ser uma população de baixo 
nível socioeconômico, as justificativas apresentadas para a maior prevalência nos menores de 24 meses são mais freqüentes. A prevalência de $83,1 \%$ foi superior à encontrada na maioria dos estudos analisa$\operatorname{dos}^{1,7,13-15}$, coincidente somente com o de Brunken e col. ${ }^{4}$.

Quando comparado com o estudo de Monteiro e col. ${ }^{1}$, realizado no município de São Paulo em crianças de 0 a 59 meses no período de 1995/1996, a prevalência de anemia em crianças freqüentadoras de creches públicas foi 31,8\% superior. Estratificando por faixa etária, a diferença entre os dois estudos apresenta maior amplitude entre os maiores de 24 meses $(41,7 \%)$. Os dados deste estudo podem apontar um aumento da prevalência de anemia ferropriva entre crianças pré-escolares (2 a 6 anos). Não é possível confirmar esta hipótese, pois para isso seria necessário um estudo de tendência temporal em uma mesma população, mas ela seria plausível com a realidade dos programas públicos de combate a anemia que priorizaram, até o presente momento, crianças menores de 24 meses e gestantes.

Nesta população, a desnutrição oculta, ou seja, o déficit nutricional de micronutrientes no qual se insere a anemia ferropriva, é mais prevalente que a desnutrição energético-proteíca (7,0\%). A faixa etária é um fator determinante comum às duas deficiências nutricionais ${ }^{15}$.

A idade materna foi a variável que, além da idade da criança, permaneceu no modelo final. Silva e col. ${ }^{5}$ e Uchimura e col. ${ }^{16}$ também verificaram associação estatística entre idade materna e anemia infantil. Uchimura e col. ${ }^{16}$ destacam a competição nutricional entre a mãe adolescente e o feto, podendo gerar crianças prematuras ou com retardo de crescimento, com baixa reserva de ferro ao nascer. No entanto, na população estudada, a ocorrência de mães adolescentes foi pequena $(4,8 \%)$. A explicação mais convincente desta associação seria aquela proposta por Silva e col. ${ }^{5}$ que consideram que mães mais jovens são mais imaturas e inexperientes quanto aos cuidados com a criança.

A variável selecionada para identificar a condição socioeconômica foi a escolaridade do chefe de família, que não foi estatisticamente significante. Até mesmo a escolaridade do responsável pela criança que pode, além da condição socioeconômica, presumir uma utilização mais eficiente da renda e dos serviços públicos, não foi estatisticamente significante. Estas associações provavelmente foram influenciadas pela homogeneidade da amostra em relação às condições socioeconômicas.

O peso ao nascer e a prematuridade são fatores importantes na gênese da anemia, já que são determinantes do estoque de ferro da criança até os primeiros seis meses de vida, e o crescimento mais acelerado no primeiro ano de vida faz com que esta doença se instale especialmente nesta fase $\mathrm{f}^{2,17}$. A ausência de associação da anemia com baixo peso ao nascer e prematuridade no presente estudo pode ser atribuída ao reduzido número de crianças nestas condições, principalmente no primeiro e segundo ano de vida (baixo peso = 11 crianças e prematuros $=8$ crianças). $\mathrm{O}$ viés de memória também pode ter prejudicado a confiabilidade destas variáveis, já que estes dados foram questionados aos responsáveis. Considerando que esta é uma população carente e a maioria das mães possui mais de um filho, recordar exatamente o peso ao nascer e o tempo de gestação em semanas é difícil e acabam respondendo a questão sem certeza da verdadeira resposta.

Segundo a Organização Mundial de Saúde, quando a prevalência da anemia é alta (acima de $40 \%$ ), a causa mais comum é a deficiência de ferro dietético, dado o baixo consumo de alimentos fontes desse micronutriente ou a alta ingestão de inibidores da absorção do ferro ${ }^{10}$. Considerando que as crianças permanecem aproximadamente 8 horas por dia na instituição, com 5 refeições diárias planejadas, mesmo que indiretamente, por profissionais da área de nutrição, a explicação 
para a prevalência tão alta de anemia não é tão evidente.

O baixo nível socioeconômico desta população os torna mais vulneráveis ao hábito alimentar inadequado no ambiente familiar e às infecções parasitárias e respiratórias, podendo comprometer o apetite e a absorção de nutrientes, variáveis não estudadas no presente estudo. Entre os menores de 24 meses, também é importante ressaltar a baixa aceitação de alimentos fonte de ferro, principalmente aqueles que possuem ferro na forma heme ${ }^{2,17}$.

Por ser grupo de risco, especialmente pela faixa etária e nível socioeconômico das crianças atendidas em creches públicas, maior atenção deve ser dada a esta população. Adequado pré-natal, incentivo ao aleitamento materno, correta introdução de alimentos, suplementação profilática com sulfato ferroso após seis meses de idade e fortificação de alimentos presentes no hábito alimentar brasileiro e de fácil acesso a toda população são exemplos de ações de combate à anemia.

Há uma variedade de estudos que avaliam a eficácia de produtos fortificados com ferro, como o proposto por CardosoSantiago e col. ${ }^{18}$, que desenvolveram um alimento contendo pulmão bovino, parte do boi pouco utilizado na culinária brasileira, porém com alto valor nutricional, que reduziu a prevalência de anemia em crianças freqüentadoras de creches ${ }^{19}$.

Desde 2002, ou seja, um ano antes deste estudo ser realizado, foi aprovada pelo governo federal brasileiro a resolução sobre a obrigatoriedade da fortificação das farinhas de trigo e milho com ferro e ácido fólico ${ }^{2}$, cuja implantação para todo o Brasil ocorreu em junho de 2004. Esta fortificação não atingiria crianças menores de 24 meses, principal grupo de risco para anemia, já que este não é um alimento muito freqüente na alimentação deste grupo etário. Desta maneira, em maio de 2005, foi instituído o Programa Nacional de Suplementação de Ferro (PNSF), que consiste na suplementação profilática medicamentosa para crianças de 6 a 18 meses $^{21}$.

Com essas intervenções é esperado que a prevalência de anemia diminua nesta população já que a maior parte da alimentação das crianças é garantida pelas instituições. É de suma importância o monitoramento e a avaliação dos diversos programas federais, estaduais e municipais que têm como objetivo o combate a esta deficiência nutricional.

\section{Referências}

1. Monteiro CA, Szarfarc SC, Mondini L. Tendência secular da anemia na infância na cidade de São Paulo (19841996). Rev Saúde Pública 2000; 34(6 Supl): 62-72.

2. Silva LSM, Giuglian ERJ, Aerts DRGC. Prevalência e determinantes de anemia em crianças de Porto Alegre, RS, Brasil. Rev Saúde Pública 2001; 35(1): 66-73.

3. Osório MM. Fatores determinantes da anemia em crianças. J Pediatr 2002; 78(4): 269-78.

4. Brunken GS, Guimarães LV, Fisberg M. Anemia em crianças menores de 3 anos que freqüentam creches públicas em período integral. J Pediatr 2002; 78(1): 50-6.

5. Silva DG, Franceschini SCC, Priore SE, Ribeiro SMR, Szarfarc SC, Souza SB, et al. Anemia ferropriva em crianças de 6 a 12 meses atendidas na rede pública de saúde do município de Viçosa, Minas Gerais. Rev Nutr 2002; 15(3):01-8
6. Oliveira RS, Diniz AS, Benigna MJC, Silva SMM, Lola MM, Gonçalves MC, et al. Magnitude, distribuição espacial e tendência da anemia em pré-escolares da Paraíba. Rev Saúde Pública 2002; 36(1):6-32.

7. Neuman NA, Tanaka OY, Szarfarc SC, Guimarães PRV, Victora CG. Prevalência e fatores de risco para anemia no Sul do Brasil. Rev Saúde Pública 2000; 34(1):6-63.

8. Bueno MB, Marchioni DML, Fisberg RM. Evolução nutricional de crianças atendidas em creches públicas no município de São Paulo. Rev Panam Salud Pública 2003; 14(3): 165-70.

9. Lohman TG, Roche AF, Martorell R. Anthropometric standardization reference manual. Champaign: Human Kinetics Books; 1988. 
10. DeMayer E, Dallman P, Gurney JM, Hallberg L, Sood SK, Srikantia SG. Preventing and controlling iron deficiency anaemia through primary health care: a guide for health administrators and programme managers. Geneva: World Health Organization; 1989.

11. Almeida CAN, Ricco RG, Ciampo LA, Souza AM, Pinho AP, Oliveira JED. Fatores associados a anemia por deficiência de ferro em crianças pré-escolares brasileiras. JPediatr 2004; 80(1): 229-34.

12. Miranda AS, Franceschini SCC, Priore SE, Euclydes MP, Araújo RMA, Ribeiro SMR, et al. Anemia ferropriva e estado nutricional de crianças com idade de 12 a 60 meses do município de Viçosa, MG. Rev Nutr 2003; 16(2): 163-9.

13. Osorio MM, Lira PI, Batista-Filho M, Ashworth A. Prevalence of anemia in children 6-59 months old in the state of Pernambuco, Brazil. Rev Panam Salud Pública. 2001;10(2): 101-7.

14. Nogueira-de-Almeida CA, Ricco RG, Ciampo LAD, Souza AM, Dutra-de-Oliveira JE. Growth and hematological studies on Brazilian children of low socioeconomic level. Arch Latinoam Nutr. 2001;51(3): 230-5.

15. Fisberg RM, Marchioni DML, Cardoso MRA. Estado nutricional e fatores associados ao déficit de crescimento de crianças freqüentadoras de creches públicas do Município de São Paulo, Brasil. Cadernos de Saúde 2004; 20(3): 812-7.

16. Uchimura TT, Szarfarc SC, Latorre MRDO, Uchimura NS, Souza SB. Anemia e peso ao nascer. Rev Saúde Pública 2003; 37(4): 397-403
17. Coutinho, GGPL Goloni-Bertollo, EM; Bertelli, ECP. Iron deficiency anemia in children: a challenge for public health and for society. Sao Paulo Med J2005; 123(2): 8892.

18. Cardoso-Santiago, R.A.C. Moreira-Araújo, R.S.R., Pinto e Silva, M.E.M. \& Arêas, J.A.G. The potencial of extruded chickpea, corn and bovine lung for malnutrition programs. Innovative Food Sci. Emmerging Technol. 2001; 2/3:203-9.

19. Moreira-Araújo, R.S.R.; Araújo, M.A.M.; Silva, A.M.S.E.; Carvalho, C.M.R.; Arêas, J.A.G.. Impacto de salgadinho de alto valor nutritivo na situação nutricional de crianças de creches municipais de Teresina-PI. Nutrire 2002, 23: 7-21.

20. BRASIL. Resolução - RDC n³44, de 13 de dezembro de 2002. Regulamento técnico para a fortificação das farinhas de trigo e das farinhas de milho com ferro e ácido fólico. Diário Oficial da União, Brasília, 18 de dezembro de 2002.

21. BRASIL. Portaria $n^{\circ} 730$, de 13 de maio de 2005. Institui o Programa Nacional de Suplementação de Ferro, destinado a prevenir a anemia ferropriva e dá outras providências. Diário Oficial da União, Brasília, 17 de maio de 2005 .

recebido em: 03/07/06 versão final reapresentada em: 21/09/06 aprovado em: 25/09/06 